Bundesgesundheitsbl 2011 · 54:980-980 DOI 10.1007/s00103-011-1350-x

(c) Springer-Verlag 2011

Mitteilungen des Arbeitskreises Blut des Bundesministeriums für Gesundheit

\title{
Blutversorgung bei Notfällen in Einrichtungen der Krankenversorgung
}

\author{
Bei der 71. Sitzung des Arbeitskreises Blut \\ am 21.03.2011 wurde folgende Stellungnahme \\ (S 10) verabschiedet:
}

er Arbeitskreis Blut sieht ein Problem in der notfallmäßigen Blutversorgung der Patienten in klinischen Einrichtungen, wenn diese vor Ort kein immunhämatologisches Labor und/oder Blutdepot zur Verfügung haben.

Der Arbeitskreis Blut empfiehlt daher den Ärztekammern, im Rahmen der Überwachung des Qualitätssicherungssystems bei der Anwendung von Blutprodukten (Kap. 1.6 Hämotherapie-Richtlinien) besonders darauf zu achten, dass in Einrichtungen der Krankenversorgung angemessene Bedingungen gewährleistet sein müssen:

1. Im Rahmen von Notfalltransfusionen ist die Transfusion ungekreuzter Erythrozytenkonzentrate der Blutgruppe 0 als so genannte Universalkonserven auf ein Minimum zu begrenzen.

2. Eine zeitgerechte Versorgung mit serologisch verträglichen $\mathrm{AB}$ 0/Rh-Faktorgleichen Erythrozytenkonzentraten muss möglich sein.
Ref.: Kiefel (Hrsg): Transfusionsmedizin und Immunhämatologie - Grundlagen, Therapie, Methodik, 4. Auflage 2010, Springer-Verlag, Berlin.

\section{Für den Arbeitskreis Blut}

Prof. Dr. R. Burger, Vorsitzender

Dr. R. Offergeld, Geschäftsführerin 